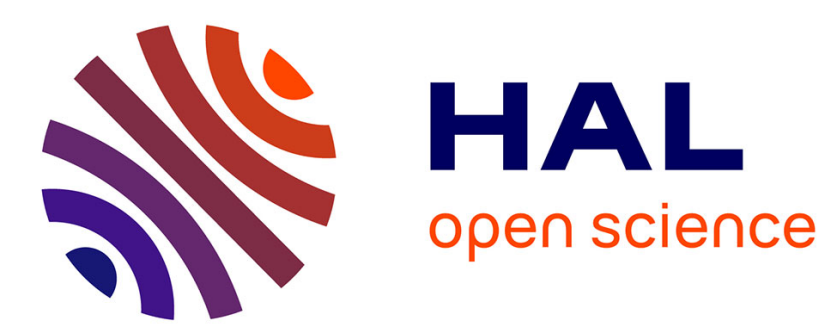

\title{
Thermomechanics of Cosserat medium: Modeling Adiabatic Shear Bands in Metals
}

\author{
Raffaele Russo, Samuel Forest, Franck Andrés Girot mata
}

\section{To cite this version:}

Raffaele Russo, Samuel Forest, Franck Andrés Girot mata. Thermomechanics of Cosserat medium: Modeling Adiabatic Shear Bands in Metals. Continuum Mechanics and Thermodynamics, In press, 10.1007/s00161-020-00930-z . hal-02966130

\section{HAL Id: hal-02966130 \\ https://hal.science/hal-02966130}

Submitted on 13 Oct 2020

HAL is a multi-disciplinary open access archive for the deposit and dissemination of scientific research documents, whether they are published or not. The documents may come from teaching and research institutions in France or abroad, or from public or private research centers.
L'archive ouverte pluridisciplinaire HAL, est destinée au dépôt et à la diffusion de documents scientifiques de niveau recherche, publiés ou non, émanant des établissements d'enseignement et de recherche français ou étrangers, des laboratoires publics ou privés. 


\title{
Thermomechanics of Cosserat medium: Modeling Adiabatic Shear Bands in Metals
}

\author{
Raffaele Russo • Samuel Forest • Franck \\ Andrés Girot Mata
}

Received: date / Accepted: date

\begin{abstract}
During most metal manufacturing processes, the medium deforms by generating large quantities of plastic strain at relatively high strain rates, inevitably inducing rises in temperature. Metals characterized by low thermal conductivity properties might locally retain high temperatures, consequently undergoing thermal softening. The classical balance laws governing the continuum equilibrium show severe mesh sensitivity if they were numerically discretized through Finite Element Methods. Furthermore, the plastic deformation tends to localize in narrow areas whose characteristic length is comparable to grain size, thereby requiring the adoption of theories able to predict sizeeffects. In this manuscript we demonstrate that the Cosserat medium is able to overcome these issues related to manufacturing processes simulation. We first provide a thermodynamically-consistent description of the Cosserat medium, and then we propose a method to calibrate the two additional characteristic lengths introduced by the Cosserat medium description by enriching the model with the TANH stress flow rule under adiabatic conditions.
\end{abstract}

Keywords Cosserat medium · Thermodynamics · Elastoviscoplasticity · Characteristic length · Adiabatic Shear Band

Raffaele Russo

Department of Mechanical Engineering, Faculty of Engineering, University of the Basque Country, 48013 Bilbao, Spain.

Centre des Matériaux, Mines-ParisTech, 91003 Evry Cedex, France.

E-mail: raffaele.russo@ehu.eus

Samuel Forest

Centre des Matériaux, Mines-ParisTech, CNRS UMR 7633, PSL Research University, BP 87, 91003 Evry Cedex, France.

Franck Andrés Girot Mata

Department of Mechanical Engineering, Faculty of Engineering, University of the Basque Country, 48013 Bilbao, Spain.

IBERBASQUE, Basque Foundation for Science, Bilbao, Spain. 


\section{Introduction}

Manufacturing processes of metallic materials can be found in almost every engineering field, e.g. aerospace, naval and civil. Performing the numerical simulations of the processes, however, still represents a big challenge in many aspects. The material, in fact, during the process, experiences prohibitive deformation fields, consequently reaching very high temperatures, close to the material melting point. From the physical standpoint, a properly-adapted model is necessary to obtain results from the simulation that would describe the real medium development.

During machining, for example, the metal is shaped into its final form through removal of small quantities of material, which is shredded from the final piece through shear deformation mainly. The material locally experiences high plastic deformation, which induce, if the adiabatic condition is met (the thermal diffusion is characterized by a lower velocity than heat production rate), to locally retain high temperatures, thus leading to material thermalsoftening. Upon material softening, a severe mesh dependence is experienced whether these equations were to be solved through Finite Element Modeling (FEM) $[15,11,51]$. On top of that, an accurate, thermodynamically-consistent material description must be employed to correctly capture the thermal softening induced by high plastic deformation $[2,32,13,53,40]$. Furthermore, the fact that during these processes the deformation is prone to localize in narrow areas makes it necessary to adopt numerical models which are able to predict size-effects $[17,21,56,28,54,35,39,42,48]$.

Since the processes of interest are highly characterized by shear deformation, the medium undergoes large material rotations at the locations where the deformation localizes. This line of reasoning, coupled with the fact that the deformations are localized in areas whose size is comparable with the grain size, led us think that the rotations experienced by the micro-structure, whose effects are normally neglected during homogeneous deformation, would assume a major role in characterizing the material response during these processes. The medium description which fits with this description is the Cosserat theory [14].

The Cosserat theory finds many applications in geomechanics, because the mechanical behavior of the soil, being a granular material at macroscopic level, is characterized by a size effects already at macroscopic level $[37,50,43]$. However, besides geomechanics, the Cosserat model has also been extensively used both for homogeneous metal materials [22,4] and polycrystal materials [18, 38, 3,27 , and many authors focused on different aspects of the Cosserat medium description. Steinmann and Willam investigated on the localization properties of the Cosserat model in elasto-plastic materials under infinitesimal deformations in case the loss of ellipticity was caused by negative material tangent operator [47]. De Borst and Sluys demonstrated that the presence of an internal length in a von Mises plastic model formulated for the Cosserat medium can be used to avoid mesh dependency when localization phenomena were simulated [5]. Most recently, Sabet and de Borst demonstrated that the Cosserat 
medium description retains ellipticity also in case the material model is characterized by a non-associated rate-independent plastic flow [45]. Kratochvíl et al. investigated over the characteristic lengths introduced by the Cosserat medium description from a physical point of view, by relating the Cosserat wryness to the Nye's dislocation tensor, thus interpreting the wryness tensor as a measure of geometrically necessary dislocations [31]. Khoei et al. drew a distinction between torsional and bending characteristic lengths, and they investigated the effect of these lengths on the shear bandwidth during different localization processes [29]. Many lines of work can also be found in literature proposing a thermodynamically-consistent model of the Cosserat kinematics $[22,4,19,20,43]$.

The problems related with metal manufacturing simulations are already well known issues, and previous researches adopted a strain gradient theory approach to overcome them $[52,26,28,44]$, however, to the best of of the author's knowledge, no research has been conducted so far on the application of the Cosserat medium for metal manufacturing simulations. Therefore, in this manuscript, the authors aim to cover this gap by providing a thermodynamically-consistent Cosserat medium description, which would be able to predict size effects even for relatively high temperatures, while retaining a compatible physical description and without loss of ellipticity. Furthermore, via the provided framework, it would be possible to unambiguously and uniquely correlate plastic deformations with temperature variations, allowing the calibration procedure to be performed through comparison of thermal fields.

In order to reproduce the same phenomena occurring in manufacturing processes, the Hat-Shaped Specimen [41] was chosen as benchmark test. The combination of specific load case and specimen geometry drives the shear deformation to localize in a narrow band that takes the name of Adiabatic Shear Band (ASB). The same ASB develops during machining operations [55, 9, 34, $36,52]$. Regarding the material model to be plugged in the Cosserat framework, the Johnson-Cook (JC) model is widely used in machining simulations [41,33, $12,8,23]$, however, it underestimates the softening effect on the flow stress [23]. Many models have been derived from the JC to overcome this issue, and we decided to use the TANH model among the others [9].

The next section provides the description of the kinematics, energetic and thermodynamic aspects of the Cosserat model. The choices of the specific form of the Helmholtz free energy and plastic models are given in Sections 2.2 and 3.2 respectively. The description of the selected benchmark test geometry and load conditions are given in Section 3. Finally, in Sections 3.3 and 3.4 the methodology used to calibrate the additional characteristic lengths introduced by the Cosserat medium are proposed. 


\section{Notation and Recalls of Tensorial Algebra}

Einstein's convention is employed in the whole manuscript. Tensors are indicated through compact notation or index notation, regardless of capital or not-capital letters:

$$
\begin{aligned}
& \underline{\boldsymbol{a}} \Longrightarrow a_{i} \text {; } \\
& \underset{\sim}{a} \Longrightarrow a_{i j} \text {; } \\
& \underset{\boldsymbol{a}}{ } \Longrightarrow a_{i j k} \text {; } \\
& \underset{\approx}{a} \Longrightarrow a_{i j k l} \text {; }
\end{aligned}
$$

The outer product is indicated with $\otimes$ and it operates in the following manner:

$$
\underset{\sim}{\mathbf{A}} \otimes \underset{\sim}{\mathbf{B}}=\underset{\approx}{\mathbf{C}} \Longrightarrow A_{i j} B_{k l}=C_{i j k l} ;
$$

Single and double contraction operators are respectively indicated as follows:

$$
\begin{aligned}
& \underset{\mathbf{A}}{\underline{\mathbf{B}}}=\underset{\approx}{\mathbf{C}} \Longrightarrow A_{i j k} B_{k l m}=C_{i j l m} ; \\
& \underset{\mathbf{A}}{\simeq}: \underset{\mathbf{B}}{\underline{\mathbf{C}}}=\underset{\sim}{\mathrm{C}} \Longrightarrow A_{i j k} B_{j k l}=C_{i l} ;
\end{aligned}
$$

Divergence and gradient operators are represented as:

$$
\begin{gathered}
\underset{\sim}{\mathbf{A}} \otimes \underline{\boldsymbol{\nabla}}=\underline{\mathbf{B}} \Longrightarrow \frac{\partial A_{i j}}{\partial x_{k}}=B_{i j k} ; \\
\underset{\sim}{\mathbf{A}} \cdot \underline{\nabla}=\underline{\mathbf{B}} \Longrightarrow \frac{\partial A_{i j}}{\partial x_{j}}=B_{i} ;
\end{gathered}
$$

where $x_{i}$ is the $i$-th Cartesian coordinate of an Eulerian space. When handling with skew-symmetric second order tensors, it might be easier to define the first order tensor associated to it as:

$$
\underline{\mathbf{A}}=-\frac{1}{2} \underline{\mathbf{\epsilon}}: \stackrel{\mathbf{B}}{\sim}
$$

where $\underset{\simeq}{\boldsymbol{\epsilon}}$ is the Levi-Civita permutation symbol.

\section{Cosserat Model}

\subsection{Kinematics and Balance Laws}

The Cosserat model [14] enhances the Classical Continuum Mechanics (CM) model by introducing three additional degrees of freedom, being the microstucture infinitesimal rotation around the three axis. The degrees of freedom of the continuum can then be written as:

$$
\left\{u_{i}, \theta_{i}\right\}, \quad i=1,2,3
$$


where $u_{i}$ is the spacial displacement along the $i$-th direction, and $\theta_{i}$ is the micro-structural rotation around the $i$-th axis. The superscript $\times$ indicates the fact that the quantity is a pseudo-vector. As function of these degrees of freedom, the deformation measures in the Cosserat medium are defined as:

$$
\begin{gathered}
\stackrel{\mathbf{e}}{\sim}=\underline{\mathbf{u}} \otimes \underline{\boldsymbol{\nabla}}-\underline{\Theta} ; \\
\stackrel{\mathbf{k}}{\sim}=\underline{\boldsymbol{\theta}} \otimes \underline{\boldsymbol{\nabla}} ;
\end{gathered}
$$

where the nabla operator $\boldsymbol{\nabla}$ is referring to the initial frame of reference and $\boldsymbol{\Theta}$ is the skew-symmetric second order tensor associated with the pseudo-vector $\underline{\theta}$ :

$$
\stackrel{\Theta}{\sim}=\underline{\underline{\epsilon}} \cdot \underline{\theta}
$$

The first deformation measure, e, is commonly referred to as Cosserat deformation, while the second, $\mathbf{k}$, is addressed as Cosserat wryness. It must be noted here that the former incorporates the difference between material infinitesimal rotation and micro-structure rotation in addition to the strain measure usually used in linearized (small deformation) $\mathrm{CM}$ as can be seen in the following:

$$
\underline{\mathbf{e}}=\operatorname{sym}(\underline{\mathbf{u}} \otimes \underline{\boldsymbol{\nabla}})+\operatorname{skew}(\underline{\mathbf{u}} \otimes \underline{\boldsymbol{\nabla}})-\underline{\Theta} ;
$$

where sym and skew indicate the symmetric and the skew-symmetric parts of a tensor respectively. The balance laws governing the Cosserat medium equilibrium can be derived by explicitly expressing the internal and external power related to the deformation measures defined in Eq. (3) and (4):

$$
P^{(i)}=\int_{\Omega}(\underset{\sim}{\sigma}: \underset{\sim}{\dot{\mathbf{e}}}+\boldsymbol{\mu}: \underset{\sim}{\dot{\mathbf{k}}}) \mathrm{d} \Omega
$$

where $\underset{\sim}{\sigma}$ is the power conjugate to the Cosserat strain rate and $\boldsymbol{\mu}$ is the power conjugate to the Cosserat wryness rate; in literature they are commonly referred to as stress and couple stress tensors. It is important to highlight here that due to the not-symmetric nature of the Cosserat strain and Cosserat wryness, both the stress and couple stress tensors are not symmetric as well. Equivalently, the external power can be defined as:

$$
P^{(e)}=\int_{\Omega}(\underline{\mathbf{f}} \cdot \underline{\dot{\mathbf{u}}}+\underline{\mathbf{c}} \cdot \underline{\dot{\theta}}) \mathrm{d} \Omega+\int_{\partial \Omega}(\underline{\mathbf{t}} \cdot \underline{\dot{\mathbf{u}}}+\underline{\mathbf{m}} \cdot \underline{\dot{\theta}}) \mathrm{d} S
$$

where $\underline{\mathbf{f}}$ is the body force per unit volume conjugate with displacement, $\underline{\mathbf{c}}$ is the body couple per unit volume conjugate with micro-structure rotation, $\underline{\mathbf{t}}$ is the surface traction conjugate with displacement, and finally $\underline{\mathbf{m}}$ is the surface couple per unit surface conjugate with micro-structure rotation. The internal power can be equivalently written as: 


$$
\begin{array}{r}
\int_{\Omega}(\underset{\sim}{\boldsymbol{\sigma}}: \underset{\sim}{\dot{\mathbf{e}}}+\underline{\mu}: \underset{\sim}{\dot{\mathbf{k}}}) \mathrm{d} \Omega=\int_{\Omega}[2 \underline{\boldsymbol{\sigma}} \cdot \underline{\dot{\theta}}-\underline{\dot{\mathbf{u}}} \cdot(\underset{\sim}{\underset{\boldsymbol{\sigma}}{\sim}} \cdot \underline{\boldsymbol{\nabla}})-\underline{\dot{\theta}} \cdot(\underline{\boldsymbol{\mu}} \cdot \underline{\boldsymbol{\nabla}})] \mathrm{d} \Omega+ \\
\\
\int_{\partial \Omega}[\underline{\dot{\mathbf{u}}} \cdot \underset{\sim}{\boldsymbol{\sigma}} \cdot \underline{\mathbf{n}}+\underline{\dot{\theta}} \cdot \underline{\boldsymbol{\mu}} \cdot \underline{\mathbf{n}}] \mathrm{dS} ;
\end{array}
$$

where the vector $\underline{\boldsymbol{\sigma}}$ is the axial vector associated with the skew-symmetric part of the stress tensor $\underset{\boldsymbol{\sigma}}{\sim}$ as in Eq. 1, and $\underline{\mathbf{n}}$ is the unit vector normal to the outer surface of the domain $\Omega$. The equality between internal and external power can be expressed in its weak form as:

$$
\begin{aligned}
\int_{\Omega} \underline{\dot{\mathbf{u}}} \cdot[(\underset{\sim}{\boldsymbol{\sigma}} \cdot \underline{\boldsymbol{\nabla}})+\underline{\mathbf{f}}] \mathrm{d} \Omega+\int_{\Omega} \underline{\dot{\theta}} \cdot[2 \underline{\boldsymbol{\sigma}}+\underline{\mathbf{c}}+(\underline{\boldsymbol{\mu}} \cdot \underline{\boldsymbol{\nabla}})] \mathrm{d} \Omega+ \\
\quad+\int_{\partial \Omega} \underline{\dot{\mathbf{u}}} \cdot[\underline{\mathbf{t}}-\underset{\sim}{\boldsymbol{\sigma}} \cdot \underline{\mathbf{n}}] \mathrm{d} \mathrm{S}+\int_{\partial \Omega} \underline{\dot{\theta}} \cdot[\underline{\mathbf{m}}-\underline{\boldsymbol{\mu}} \cdot \underline{\mathbf{n}}] \mathrm{d} \mathrm{S}=0 ;
\end{aligned}
$$

from which, given the arbitrariness of $\underline{\dot{u}}$ and $\underline{\dot{\theta}}$, the strong forms of the linear and angular momentum balances can be derived as:

$$
\begin{gathered}
\stackrel{\boldsymbol{\sigma}}{\sim} \cdot \underline{\boldsymbol{\nabla}}+\underline{\mathbf{f}}=\underline{\mathbf{0}} ; \\
\underline{\mu} \cdot \underline{\boldsymbol{\nabla}}+2 \underline{\boldsymbol{\sigma}}+\underline{\mathbf{c}}=\underline{\mathbf{0}} ;
\end{gathered}
$$

which are vectorial partial differential equations in the variables $\underline{\mathbf{u}}$ and $\underline{\theta}$, bounded by:

$$
\begin{gathered}
\underset{\sim}{\sigma} \cdot \underline{\mathbf{n}}=\underline{\mathbf{t}} ; \\
\underline{\mu} \cdot \underline{\mathbf{n}}=\underline{\mathbf{m}} ;
\end{gathered}
$$

\subsection{Clausius-Duhem inequality}

The material behavior can be explicitly stated in terms of stress-strain relationship or by expressing the Helmholtz free energy and resorting to the Clausius-Duhem inequality. Given that our objective is to characterize the energetic behavior of the Cosserat medium, in this work we employ the second method. The following decompositions of strain and wryness are adopted:

$$
\begin{aligned}
& \underset{\sim}{\mathbf{e}}={\underset{\sim}{\mathbf{e}}}^{e}+{\underset{\sim}{\mathbf{e}}}^{p}+{\underset{\sim}{\mathbf{e}}}^{t h} \\
& \mathbf{k}=\underset{\sim}{\mathbf{k}} \\
& \mathbf{k}^{e}+{\underset{\sim}{p}}^{p}+{\underset{\sim}{\mathbf{k}}}^{t h}
\end{aligned}
$$

in which the relation between elastic, plastic and thermal deformation has an additive character, due to the linearization performed under small displacements and small rotations assumptions. It is then necessary to define the state variables of the medium, and in this work they are the following:

$$
\left\{{\underset{\sim}{\mathbf{e}^{e}}}^{\mathbf{k}^{e}}, T, \alpha\right\} ;
$$


namely, elastic strain, elastic wryness, temperature and a hardening variable, which can be a tensor of any rank. Based on the choice of the state variables, the Helmholtz free energy density per unit volume can be written as:

$$
\psi=\psi\left(\underset{\sim}{\mathbf{e}^{e}},{\underset{\sim}{\mathbf{k}}}^{e}, T, \alpha\right)
$$

Here a more explicit definition of the Helmholtz free energy, linearized with respect to the initial conditions $\left\{\mathbf{e}_{0}^{e}=0, \mathbf{k}_{0}^{e}=0, T_{0}, \alpha_{0}=0\right\}$, is provided in a still quite generic form. It assumes quadratic potentials for the elastic deformation measures, typical energy definition related to thermal deformations, standard energy definition related to heat exchange and an additional term concerning the stored energy relative to plastic deformations:

$$
\begin{aligned}
& \psi\left(\underset{\sim}{\mathbf{e}^{e}}, \mathbf{k}^{e}, T, \alpha\right)=\frac{1}{2}{\underset{\sim}{e}}^{e}: \underset{\approx}{\boldsymbol{\Lambda}}:{\underset{\sim}{e}}^{e}+\frac{1}{2} \mathbf{k}^{e}: \underset{\approx}{\mathbf{C}}:{\underset{\sim}{k}}^{e}-\left(T-T_{0}\right) \underset{\sim}{\mathbf{P}}:{\underset{\sim}{e}}^{e}
\end{aligned}
$$

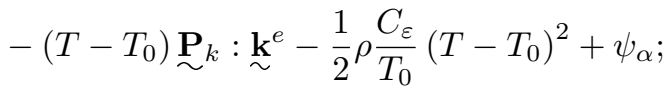

where $\underset{\approx}{\mathbf{\Lambda}}$ and $\underset{\approx}{\mathbf{C}}$ are the fourth-order elastic stiffness tensors, relating stressstrain and couple stress-wryness respectively, $\underset{\sim}{\mathbf{P}}$ and $\underset{\sim}{\mathbf{P}_{k}}$ are second order tensors relating thermal variations with elastic expansions or wrynesses respectively, $C_{\varepsilon}$ is the specific heat capacity, $\rho$ is the density and $\psi_{\alpha}$ is the part of the Helmholtz free energy that accounts for variation in the recoverable energy of the continuum due to variation in the material yield limit, e.g. hardening or softening. Subsequently, we employ the first and second thermodynamic principles to write the Clausius-Duhem inequality and to explicitly express the dissipation of the continuum. From the first thermodynamic principle:

$$
\dot{u}=\underset{\sim}{\sigma}: \underset{\sim}{\dot{\mathbf{e}}}+\underline{\boldsymbol{\mu}}: \underset{\sim}{\dot{\mathbf{k}}}-\underline{\mathbf{q}} \cdot \underline{\boldsymbol{\nabla}}+r
$$

where $\dot{u}$ is the internal energy rate density per unit volume, not to be mistaken with the displacement rate, $\underline{\mathbf{q}}$ is the heat flux and $r$ is the heat production. The second thermodynamic principle reads:

$$
\dot{\eta}-\frac{r}{T}+\left(\frac{\underline{\mathbf{q}}}{T}\right) \cdot \underline{\nabla} \geq 0
$$

where $\dot{\eta}$ is the entropy rate density per unit volume. Considering that the Helmholtz free energy is defined as:

$$
\psi\left({\underset{\sim}{\mathbf{e}^{e}}}_{\mathbf{k}^{e}}^{e}, T, \alpha\right)=u-T \eta
$$

and by expressing its differential as:

$$
\dot{\psi}=\left[\underset{\sim}{\partial{\underset{\sim}{e}}^{e}} \dot{\mathbf{e}}^{e}+\frac{\partial \psi}{\partial \dot{\sim}^{e}} \dot{\sim}^{e}+\frac{\partial \psi}{\partial T} \dot{T}+\frac{\partial \psi}{\partial \alpha} \dot{\alpha}\right]=\dot{u}-T \dot{\eta}-\dot{T} \eta
$$


the second thermodynamic principle assumes the following form:

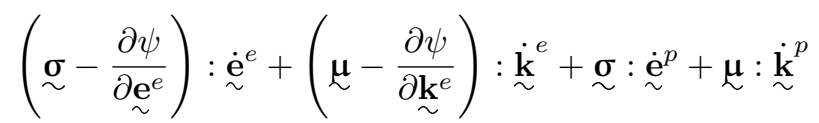

$$
\begin{aligned}
& -\left(\frac{\partial \psi}{\partial T}+\eta+\underset{\sim}{\boldsymbol{\sigma}}: \frac{\partial \mathbf{e}^{t h}}{\partial T}+\boldsymbol{\mu}: \frac{\partial \mathbf{k}^{t h}}{\partial T}\right) \dot{T}-\frac{\partial \psi}{\partial \alpha} \dot{\alpha}-\frac{\underline{q} \cdot(\boldsymbol{\nabla} T)}{T} \geq 0 ;
\end{aligned}
$$

from which, assuming that the elastic strain, elastic wryness and thermal variation are fully recoverable, the followings can be derived:

$$
\begin{aligned}
& \underset{\sim}{\boldsymbol{\sigma}}=\frac{\partial \psi}{\partial \mathbf{e}^{e}} \\
& \boldsymbol{\mu}=\frac{\partial \psi}{\partial{\underset{\sim}{\mathbf{k}^{e}}}^{e}} ; \\
& \eta=-\frac{\partial \psi}{\partial T}+\underset{\sim}{\boldsymbol{\sigma}}: \frac{\partial \mathbf{e}^{t h}}{\partial T}+\boldsymbol{\mu}: \frac{\partial \mathbf{k}^{t h}}{\partial T} ;
\end{aligned}
$$

and, by expanding Eq. (25) and (26), the following relation between thermal deformations, elastic deformations and stress/couple stress can be found:

$\underset{\sim}{\boldsymbol{\sigma}}=\frac{\partial \psi}{\partial \mathbf{e}^{e}}=\underset{\approx}{\boldsymbol{\Lambda}}:{\underset{\sim}{\mathbf{e}}}^{e}-\underset{\sim}{\mathbf{P}}\left(T-T_{0}\right)=\underset{\approx}{\boldsymbol{\Lambda}}:\left[\mathbf{e}^{e}-\underset{\approx}{\mathbf{\Lambda}}{ }^{-1}: \underset{\sim}{\mathbf{P}}\left(T-T_{0}\right)\right]=\underset{\approx}{\boldsymbol{\Lambda}}:\left[\mathfrak{\mathbf { e }}^{e}-{\underset{\sim}{ }}^{t h}\right] ;$

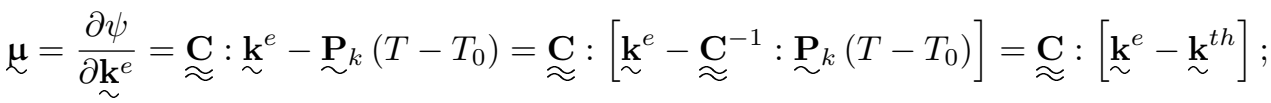

where:

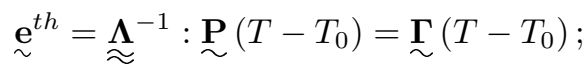

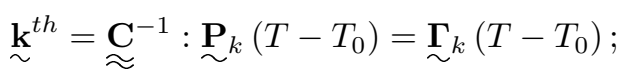

and the tensors $\underset{\sim}{\boldsymbol{\Gamma}}$ and $\underset{\sim}{\boldsymbol{\Gamma}} k$ are operators used to compute the thermal expansion/wryness. The remaining terms in Eq. (24) define the medium dissipation, here referred to as $\Pi$ :

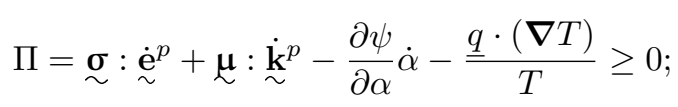

Furthermore, we can write the heat equation from Eq. (20) and (23) as:

$$
\underset{\sim}{\boldsymbol{\sigma}}: \dot{\sim}^{p}+\boldsymbol{\mu}: \dot{\sim}^{p}-\underline{\mathbf{q}} \cdot \nabla+r=\frac{\partial \psi}{\partial \alpha} \dot{\alpha}+T \dot{\eta} ;
$$


2.3 Specific Choices of the Material Model

\subsubsection{Elastic part}

From Eq. (25) and (26) it can be observed that the form of the constitutive equations of stress and couple-stress directly derives from the chosen form of the Helmholtz free energy, it is then essential to give a proper shape to this potential. The Helmholtz free energy form adopted in Eq. (19) is still quite general, and many mechanical behaviors can be modeled using this form. The most simple model used for isotropic materials can be expressed as [7]:

$$
\begin{aligned}
& \underset{\sim}{\boldsymbol{\sigma}}=\lambda \operatorname{tr}\left({\underset{\sim}{\mathbf{e}^{e}}}^{\mathbf{I}} \mathbf{\sim}+2 \mathrm{G} \operatorname{sym}\left({\underset{\sim}{\mathbf{e}^{e}}}^{\mathbf{I}}+2 \mathrm{G}_{c} \operatorname{skw}\left(\underset{\sim}{\mathbf{e}^{e}}\right) ;\right.\right. \\
& \boldsymbol{u}=\xi \operatorname{tr}\left(\sim_{\sim}^{\mathbf{k}^{e}}\right) \underset{\sim}{\mathbf{I}}+2 \beta \operatorname{sym}\left(\sim_{\sim}^{\mathbf{k}^{e}}\right)+2 \gamma \operatorname{skw}\left({\underset{\sim}{\mathbf{k}}}^{e}\right) ;
\end{aligned}
$$

where $\mathbf{I}$ is the Identity second-order tensor, and the following conditions on the material parameters must be valid:

$$
\left\{\begin{array} { l } 
{ 3 \lambda + 2 \mathrm { G } \geq 0 } \\
{ \mathrm { G } \geq 0 } \\
{ \mathrm { G } _ { c } \geq 0 }
\end{array} \quad \& \quad \left\{\begin{array}{l}
3 \xi+2 \beta \geq 0 \\
\beta \geq 0 \\
\gamma \geq 0
\end{array}\right.\right.
$$

In this constitutive model, $\lambda$ and $G$ are the usual Lamé parameters and $G_{c}$, $\xi, \beta$ and $\gamma$ are additional material coefficients. In the $2 \mathrm{D}$ case, it is customary to assume $\beta=\gamma$ for simplicity [5]. For our computations, we are prone to use relatively high values of $G_{c}$ when compared to $G$, such that the mismatch would be characterized by a stiffer response, and the value of $\operatorname{skw}\{\underline{\mathbf{u}} \otimes \underline{\boldsymbol{\nabla}}\}$ would be negligible if compared to $\operatorname{sym}\{\underline{\mathbf{u}} \otimes \underline{\boldsymbol{\nabla}}\}$. In this case, the Cosserat theory would recover the characteristics of a couple-stress theory, also known as constrained Cosserat theory [30]. There is, however, a conceptual difference between the hydrostatic term of the wryness and its deviatoric counterpart: while, in fact, the former is related to variation of the micro-structure rotation along the axis in which it is defined (torsion), the latter expresses the variation of the micro-structure rotation along the other two directions (bending), and in general these two deformations might be characterized by different responses. After a simple dimensional analysis, the following elastic characteristic length of the Cosserat medium can be defined:

$$
l_{e l}=\sqrt{\frac{\beta}{G}}
$$

To be noted that in case no simplifications were done on the constitutive behavior of the Cosserat model, other characteristic lengths were identifiable, as done in other researches [29]. 


\subsubsection{Viscoplastic part}

In the same way we defined the material response by defining the Helmholtz free energy instead of the stress-strain relation, it is possible to explicitly state the shape of the viscoplastic potential associated to the plastic deformation. A general shape of the multi-criterion Cosserat viscoplastic potential is retrieved [19] where two potentials are defined, one related to $\underset{\sim}{\mathbf{e}^{p}}$ and one related to $\underset{\sim}{\mathbf{k}}$. They can be modeled using the general viscoplastic potential form as the one proposed by Chaboche in case of standard continuum mechanics [10] :

$$
\Omega=\frac{D}{k+1}\left(\frac{\langle f(\underset{\sim}{\boldsymbol{\sigma}}, p, T)\rangle}{D}\right)^{k+1}+\frac{L}{\xi+1}\left(\frac{\left\langle g\left(\boldsymbol{\mu}, p_{k}, T\right)\right\rangle}{L}\right)^{\xi+1}
$$

where $\Omega$ is the viscoplastic potential, $D$ and $L$ are the drag stress and couple stress, $k$ and $\xi$ are parameters to be calibrated and $\langle f\rangle$ and $\langle g\rangle$ are over-stress and over-couple-stress between the Macaulay brackets. If we assume associated plasticity and explicitly give the form of $\Omega$, the plastic behavior would be completely defined. This is because the plastic strain rate and plastic wryness rate can be written as:

$$
\stackrel{\dot{\mathbf{e}}}{ }^{p}=\frac{\partial \Omega}{\partial f} \frac{\partial f}{\partial \underset{\sim}{\boldsymbol{\sigma}}}, \quad \dot{\mathbf{k}}^{p}=\frac{\partial \Omega}{\partial g} \frac{\partial g}{\partial \underline{\mu}} ;
$$

from which, the first ratios are equal to the rates of the viscoplastic multipliers:

$$
\dot{p}=\frac{\partial \Omega}{\partial f}, \quad \dot{p}_{k}=\frac{\partial \Omega}{\partial g}
$$

and the second ones define the direction of the plastic flows, being equal to the one of the associated stress/couple stress:

$$
\frac{\partial f}{\partial \underset{\sim}{\sigma}}={\underset{\sim}{\mathbf{n}_{\sigma}}}, \quad \frac{\partial g}{\partial \underset{\sim}{\mu}}={\underset{\sim}{\mathbf{n}_{\mu}}}
$$

The choice of expressing the viscoplastic potential as in Eq. (38) would impose the calibration of additional parameters related with both the contributions coming from plastic deformation and plastic wryness. However, the expression of the viscoplastic potential usually used with the Cosserat medium is simplified by assuming a single criterion, thus a single viscoplastic multiplier, which is common to both plastic deformation and plastic wryness:

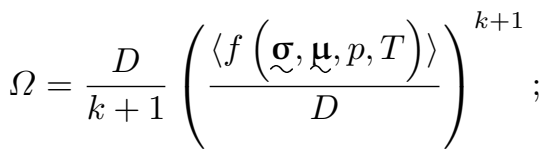

and:

$$
\dot{p}=\frac{\partial \Omega}{\partial f}, \quad \dot{\mathbf{e}}^{p}=\dot{p} \frac{\partial f}{\partial \underset{\sim}{\boldsymbol{\sigma}}}, \quad \dot{\mathbf{k}}^{p}=\dot{p} \frac{\partial f}{\partial \boldsymbol{\mu}} ;
$$


with $f$ depending both on stresses and couple stresses:

$$
f=f(\underset{\sim}{\boldsymbol{\sigma}}, \underset{\sim}{\boldsymbol{\mu}}, p, T)=\sigma_{e q}(\underset{\sim}{\boldsymbol{\sigma}}, \underset{\boldsymbol{\mu}}{)}-R(p, T) ;
$$

where $R$ represents the radius of the yield surface above which plasticity is activated. In the Cosserat framework, several equivalent stress measures can be adopted :

$$
\begin{aligned}
& \sigma_{\mathrm{eq}}=\sqrt{\frac{3}{2}\left(\mathrm{a}_{1} \boldsymbol{\sigma}^{\prime}:{\underset{\boldsymbol{\sigma}}{ }}^{\prime}+\mathrm{a}_{2}{\underset{\sim}{\boldsymbol{\sigma}}}^{\prime}:{\underset{\boldsymbol{\sigma}}{ }}^{\mathrm{T}}+\mathrm{b} \boldsymbol{\mu}: \boldsymbol{\mu}\right)} ;
\end{aligned}
$$

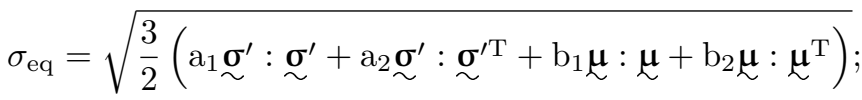

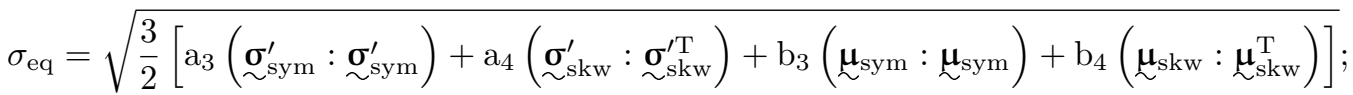

where the first one was proposed by de Borst $[5,6]$, the second one by Forest [19], and the third one is based on the invariant decomposition of a second order tensor [57]. The operator $\boldsymbol{\sigma}^{\prime}$ returns the deviatoric part of the tensor $\boldsymbol{\sigma}$. Based on the dimensional analysis of the coefficients appearing in the Equations above, and assuming that the terms $\mathrm{a}_{i}$ are equal to each other, as well as the $\mathrm{b}_{i}$ terms, the plastic characteristic length arises from the model as:

$$
l_{p}=\sqrt{\frac{\mathrm{a}}{\mathrm{b}}}
$$

The last remark regarding the completeness of the plastic model concerns the definition of the plastic part of the Helmholtz free energy, that is, $\psi_{\alpha}$ in Eq. (19). This is, in general, equal to [46]:

$$
\psi_{\alpha}=\int_{0}^{\alpha} \pi(\xi) d \xi
$$

where $\pi$ is a general stress thermodynamically associated to the internal variable $\alpha$. In most plastic evolution laws the equivalent cumulative plastic strain is used as hardening parameters, such that $\dot{p}=\dot{\alpha}$, and $\pi$ is essentially the stress thermodynamically conjugated to the equivalent cumulative plastic strain. Given the integral formulation of this term, its closed form does not necessarily exist, and it could be eventually found once the plastic flow rules are defined.

\subsubsection{Adiabatic Temperature Evolution}

The time variation of the state variables is then to be defined. Given total strain and wryness increment, the elastic stain/wryness, viscoplastic multiplier and thermal rates must be evaluated. The derivation of the adiabatic temperature rate can be found in the appendix, and it is here reported: 


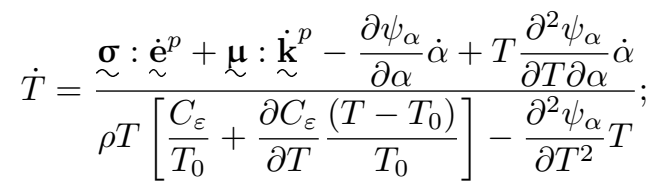

which in case of further simplification becomes:

$$
\dot{T}=\frac{\underset{\sim}{\boldsymbol{\sigma}}: \dot{\mathbf{e}}^{p}+\underset{\sim}{\sim}: \dot{\mathbf{k}}^{p}-\frac{\partial \psi_{\alpha}}{\partial p} \dot{p}}{\rho T \frac{C_{\varepsilon}}{T_{0}}} ;
$$

Note that it is possible, with this formulation, to evaluate the part of plastic work converted into adiabatic temperature increment without any additional calibration to be performed. Many researches can be found in literature where the effects of hardening/softening on the temperature increment is incorporated in a unique parameter, namely the Taylor-Quinney coefficient $\Xi$ [49], that requires calibration in different condition:

$$
\dot{T}=\frac{\Xi}{\rho C_{\varepsilon}}\left(\underset{\sim}{\sigma}: \dot{\sim}^{p}+\underline{\mu}: \dot{\sim}^{p}\right) ;
$$

By using this temperature evolution law and the evolution of the plastic deformations defined in Eq. (43), the elastic predictor can then be written as:

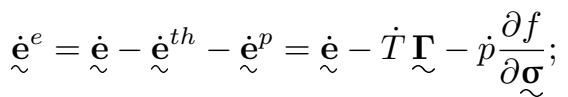

$$
\begin{aligned}
& \stackrel{\mathbf{k}}{ }^{e}=\underset{\sim}{\dot{\mathbf{k}}}-\dot{\sim}^{t h}-{\underset{\sim}{\mathbf{k}}}^{p}=\underset{\sim}{\dot{\mathbf{k}}}-\dot{T} \underset{\sim}{\boldsymbol{\Gamma}_{k}}-\dot{p} \frac{\partial f}{\partial \underset{\sim}{\mu}} ;
\end{aligned}
$$

\section{Model Implementation and Characteristic Lengths Calibration}

The model described in the previous section is here proposed to be a suitable solution for manufacturing processes simulations. The main reason is the embodied property of the model to enlarge the domain of the vectorial solution space $\{\underline{\mathbf{u}}\}$ for which the ellipticity of the PDEs is retained. In most of the plastic models employed to simulate manufacturing processes, the strain hardening effect overcompensates the thermal softening, thus the PDEs rarely experience a loss of ellipticity. However, when high temperatures are expected to develop, material softening could most likely exceed the strain hardening, but not many material models are able to properly predict the flow stress at high temperatures $\left(\approx 0.8 T_{m}\right.$-melting temperature), therefore thermal softening might not be correctly captured. The Johnson-Cook model, widely used in manufacturing processes, is known to underestimate the softening effect [23], and its implementation in a strain-gradient theory was already documented 
[52]. Due to the limitation of the JC model in capturing material softening, many modified versions model can be found in literature. Among the others, we decided to use the TANH model [9]. Both the Classical CM model and the Cosserat model have been fetched with this material description.

Therefore, acknowledging the lacking of a proper material plastic model able to predict flow stress models at high operational temperature, in this section we limit ourself to demonstrate the procedure by which the two additional Cosserat characteristic lengths might be calibrated. Given our final object of employing the Cosserat medium to simulate manufacturing processes, the calibration procedure will be performed by modeling a Hat-Shaped Specimen under compression [41]. The Hat-Shaped test is characterized by the development of an ASB inside the specimen, followed by an increase in temperature due to the high levels of localized plastic strain. Since most of the manufacturing processes that export material from the final piece are designed to do so through the development of ASBs and subsequent material removal, this example could represent a simplified condition of the one occurring during manufacturing. In fact, the calibration procedure has been performed precisely while looking at the fields developed at the ASB location.

The geometry of the specimen was taken from the work of Peirs et al. [41]. Although the same geometry and material properties were used, the plastic behavior chosen for this test is different than the one adopted by Peirs et al. The specification of the plastic model and the material properties will be assessed in Section 3.2, and aim to reproduce the behavior of the Titanium alloy Ti6Al4V. Given the axial-symmetry of the specimen, only half of the cross section has been modeled in 2D under axial-symmetric conditions.

In Figure 1 the geometry and the boundary conditions of the benchmark tests are reported. A vertical downward velocity of $5.5 \mathrm{~m} / \mathrm{s}$ was imposed at the top surface of the specimen, and the simulations lasted until a vertical negative displacement of $-0.275 \mathrm{~mm}$ was reached. The simulations have been produced using the FEM solver Z-set [1], in which the Cosserat element description and material behavior are implemented. Quadratic interpolation has been used to discretize displacement and rotation fields between nodal values, thereby quadratic elements have been used.

\subsection{Mesh-independence Simulations \& Mesh Size Choice}

One of the reasons for us to employ a Cosserat medium description is to avoid the mesh dependency developed when the material softens at high temperatures. Therefore, a preliminary verification was performed in order to be sure that the Cosserat medium description would be able to produce meshinsensitive results. Simulations of the Hat-Shaped specimen under compression were carried out using the Classical CM and the Cosserat descriptions both fetched with a plastic behavior that reproduces pure softening. For these simulations, the mechanical and thermal properties reported in Table 1 were used. In addition to the ones indicated in the table, the couple stress moduli $\beta$ and 


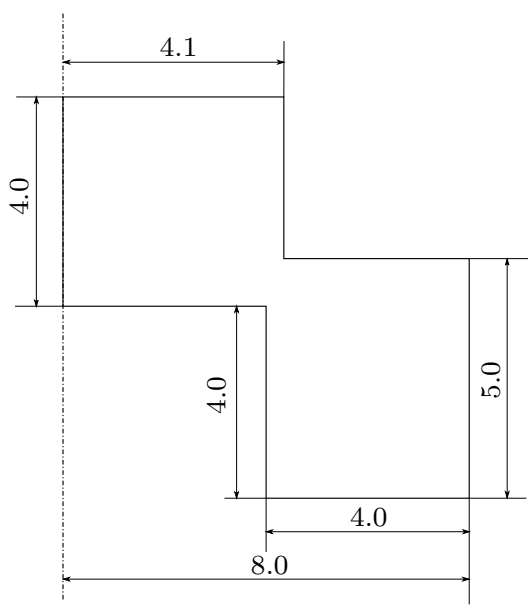

(a)

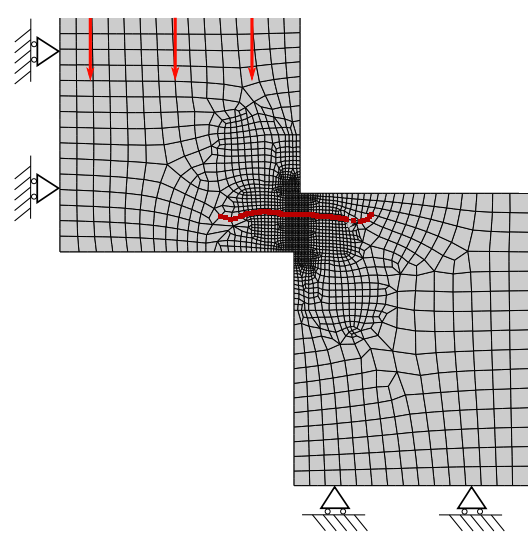

(b)

Fig. 1: Hat-Shaped Specimen [41], geometry (a) and mesh with boundary conditions (b). Units in millimeters. In (b) the cross section along the shear band is indicated with red nodes.

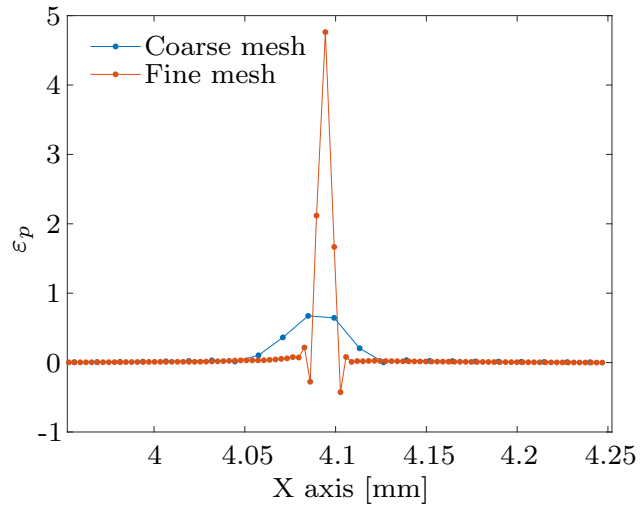

(a) Classical CM.

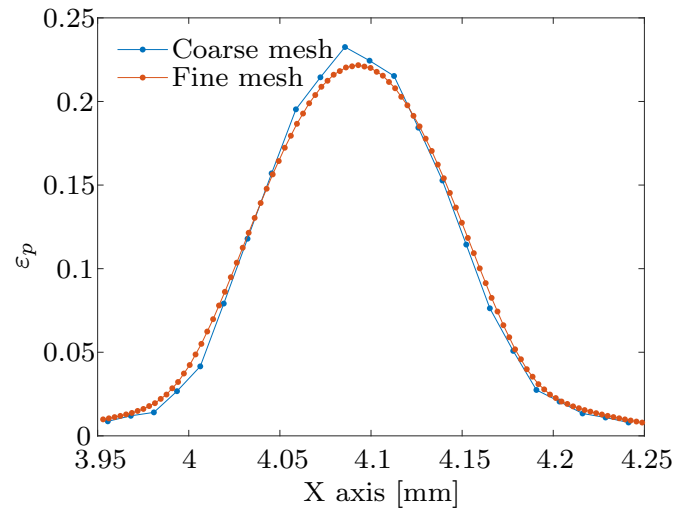

(b) Cosserat medium.

Fig. 2: Cumulative plastic distribution for different mesh sizes using Classical CM (a) and Cosserat medium (b).

$\gamma$ were both equal to $0.114 \mathrm{MPa} \cdot \mathrm{mm}^{2}$. Although this model might not be descriptive of the behavior of any material, this numerical exercise allow us to verify the mesh independence in the most sever condition of pure softening. The flow stress adopted for this exercise reads:

$$
R(p)=R_{0}+H p
$$


where $\mathrm{H}$ is the softening modulus, which was set equal to - $30 \mathrm{MPa}$. As it can be seen from Eq. (55), the equivalent cumulated plastic strain in this case is causing the same effect that is usually induced by the temperature on the flow stress. According to Eq. (55), the material softening is induced whenever plastic strain cumulates, while normally the softening is a thermally-activated phenomena. The same simulation was performed for two different mesh size, a finer mesh of $20 \mu \mathrm{m} \mathrm{x} 15 \mu \mathrm{m}$ and a coarser mesh of double the size for both the Classical CM and the Cosserat medium, and the plastic distributions across the ASB were compared. In Figure 2 the comparison is appreciable. The equivalent plastic strain distribution has been plotted across the ASB. While the Classical CM description exhibits a strong mesh-dependency, the Cosserat medium does not show such dependency. More discussion related to the differences in cumulative plastic distribution between Classical CM and Cosserat medium will be provided in the Section 3.3, but this results clearly shows that the Cosserat medium can retain mesh independence results under conditions for which the Classical CM cannot.

\section{Mesh Size}

In order to ensure the mesh size to be smaller than the elastic characteristic length of the model, a preliminary investigation was made to find the characteristic length for which the simulations would converge. In this analysis, the assumptions that both $a_{i}$ and $b_{i}$ are equal to 0 in Eq. (46) were made. Consequently, both the deformation and the wryness are fully elastic. Under this assumptions, the Hat-Specimen under compression was simulated using an initial mesh size of $20 \mu \mathrm{m} \times 15 \mu \mathrm{m}$, and using the material properties as Table 1. The results in terms of load-displacement of the Hat-Shaped Specimen are reported in Figure 3. The values of the elastic characteristic length range from $10^{1} \mathrm{~mm}$ to $10^{-4} \mathrm{~mm}$. The curves achieve convergence already for values of the elastic characteristic length of $10^{-2} \mathrm{~mm}$, therefore suggesting the adoption of a mesh size, along the direction orthogonal to the ASB, smaller than $100 \mu \mathrm{m}$. Subsequently, using a mesh size of $40 \mu \mathrm{m} \times 30 \mu \mathrm{m}$ can be considered a safe choice. Furthermore, for all the simulations, quadratic elements are used, so that the distance between the nodes spanning the element edges is ulteriorly halved.

\subsection{Specifications of the Plastic Model}

Although the model previously described defines the plastic behavior of the medium by providing the explicit function of the plastic potential $\Omega$, most of the models used in literature are simply provided through their flow stress definition.

The flow stress used in this manuscript derives from the one developed by Calamaz et al. [9], but the dependence of the flow stress on the rate of the equivalent plastic strain has been omitted in order to simplify the model 


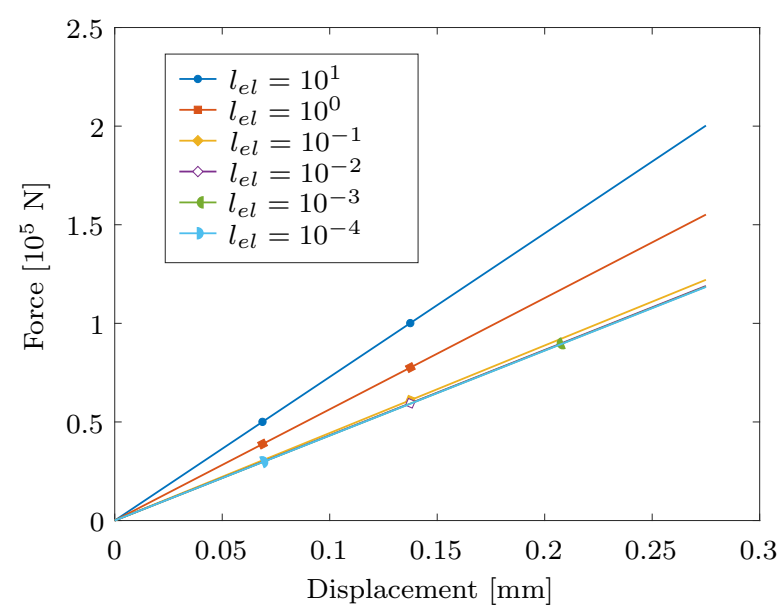

Fig. 3: Convergence analysis of the elastic characteristic length performed on the load-displacement graph of the Hat-Shaped Specimen using $b_{i}=a_{i}=0$ as assumption in Eq. (46). Values of the characteristic lengths are given in millimeters.

and focus only on strain hardening and thermal softening behaviors. This flow stress model has been used several times in literature [25,24], but it has never been coupled with the Cosserat medium description. The radius of the yield surface is defined as:

$$
R=\left[A+\frac{B p^{n}}{\exp \left(p^{a}\right)}\right]\left[1-\left(\frac{T-T_{0}}{T_{m}-T_{0}}\right)^{m}\right]\left[D+(1-D) \tanh \left(\frac{1}{(p+S)^{c}}\right)\right]
$$

where:

$$
\begin{gathered}
D=1-\left(\frac{T}{T_{m}}\right)^{d} \\
S=\left(\frac{T}{T_{m}}\right)^{b}
\end{gathered}
$$

and the flow rule of the viscoplastic multiplier $p$ follows:

$$
\dot{p}=\exp \left(\frac{\langle f\rangle}{R C}\right)-1=\exp \left[\frac{1}{C}\left(\frac{\sigma_{e q}}{R}-1\right)\right]-1 ;
$$

It must be remembered that since we are using a single viscoplastic multiplier, $\dot{p}$ depends on both plastic strain and wryness contributions. The material properties are listed in Table 1, and they belong to the Titanium alloy Ti6Al4V, and the TANH flow stress model parameters have been summarized in Table 2. 


\begin{tabular}{|c|c|c|c|c|c|c|c|c|}
\hline $\mathrm{E}[\mathrm{MPa}]$ & $\nu$ & $G_{c}[\mathrm{MPa}]$ & $\rho\left[\mathrm{kg} / \mathrm{m}^{3}\right]$ & $C_{\varepsilon}[\mathrm{J} / \mathrm{kg} \cdot \mathrm{K}]$ & $T_{0}[\mathrm{~K}]$ & $T_{m}[\mathrm{~K}]$ & $\Gamma[1 / \mathrm{K}]$ & $\Gamma_{k}[1 / \mathrm{K} \cdot \mathrm{mm}]$ \\
\hline 114000.0 & 0.3 & 114000.0 & 4428.0 & 580.0 & 293.0 & 1926.0 & $8.5 \mathrm{e}-6$ & 0.0 \\
\hline
\end{tabular}

Table 1: Elastic and thermal material properties corresponding to the Titanium allot Ti6Al4V [41].

\begin{tabular}{|c|c|c|c|c|c|c|c|c|}
\hline $\mathrm{A}[\mathrm{MPa}]$ & $\mathrm{B}[\mathrm{MPa}]$ & $\mathrm{C}$ & $\mathrm{m}$ & $\mathrm{n}$ & $\mathrm{a}$ & $\mathrm{b}$ & $\mathrm{c}$ & $\mathrm{d}$ \\
\hline 1120.0 & 667.0 & 0.0270 & 1.33 & 0.47 & 1. & 1. & 1. & 1. \\
\hline
\end{tabular}

Table 2: Visco-plastic coefficients used in the TANH model [41].

\subsection{Elastic characteristic length calibration}

In our case, since we are modeling in $2 \mathrm{D}$, there is no torsional component in the wryness $\left(\mathrm{k}_{i i}^{e}=0, i i=1,2,3\right)$, therefore the parameter $\alpha$ does not have any influence and it must not be calibrated. Throughout the calibration process the value of $G_{c}$ was set constant and imposed to be higher than the second Lame parameter (shear modulus), such that the Cosserat model would assume similar nature of a strain gradient theory. The value of $G_{c}$ was set to be equal to $114000.0 \mathrm{MPa}$. The calibration of the elastic characteristic length can be performed by simply varying the coefficient $\beta$ in Eq. (37). The Classical CM framework can be retrieved by imposing the elastic characteristic length to vanish, and in this case the deformation localizes as if it was unaffected by the "diffusing" effect induced by not-vanishing $\mathbf{k}$. The calibration procedure shall start from large values of $\beta$ and then its values should progressively reduce. Differently from the analysis done in the previous section, for the current calibration procedure only the condition $b_{1}=b_{2}=0$ was used in Eq. (46), that is, the plastic characteristic length was set equal to infinity. Consequently, the developed curvature is fully elastic, but the strain is also plastic, and $\dot{p}$ depends on the stress only in Eq. (46).

The calibration procedure could be performed by comparing data of different nature between experiments and simulations. Following the complete thermodynamic description outlined in Section 2 we could compare the temperature distribution in the ASB, especially because this can be easily measured through thermal camera or, in case the plastic deformation localizes inside the specimen, by positioning thermocouples at the location where the ASB is expected to develop. Since the magnitude of the characteristic length influences the area over which the developed deformation spreads (thickness of ASB), many thermocouples might be necessary to capture both the thermal distribution and its peak value. To be noted here that in adiabatic conditions the thermal variation is only driven by local development of plastic deformation, since there is no time for the temperature to diffuse, thus the measured temperature is a direct indication of the developed plastic deformation. Six values of the elastic characteristic length were used, from $10^{1} \mathrm{~mm}$ to $10^{-4} \mathrm{~mm}$. 
The effects of the elastic characteristic length have been investigated by looking both at the load-displacement curve and at the temperature distribution at the ASB.

As already observed in case of a hyper-elastic material model, the adoption of an elastic characteristic length, whose order of magnitude is comparable with the geometry size, extends the stiffening effect to a macroscopic level, inducing the whole system to behave stiffer. In case a plastic component is introduced in the Cosserat strain, the same behavior is observed, as inferred

by Figure 4a. It can be observed from this Figure that the over-prediction of the initial elastic stiffness is negligible for values of the characteristic length smaller or equal than $\approx 10^{-1} \mathrm{~mm}$. Furthermore, it can be appreciated from the same Figure the trend of the load-displ curve of converging toward the results produced for a Classical CM simulation for values of the characteristic length that approach zero. The curve, however, should not overlap with the one obtained from the adoption of the TANH model in a Classical CM framework because, although the curvature is providing a null energetic contribution, the part of the Cosserat strain accounting for the mismatch between macro and micro rotation is still playing a role.

In Figure 5 the thermal fields across the ASB are reported. From the Figure, it is possible to identify the effect of the characteristic length on the thermal distribution. The elastic characteristic lengths indicate the range over which the thermal field, in this case, is spreading: smaller elastic characteristic length will induce the fields to spread over smaller domains. It can be noted that the temperatures reached in the Classical CM case are lower than the ones in the Cosserat model with a characteristic length of $10^{-4} \mathrm{~mm}$, however, it should be reminded that the results of this simulation strongly depend on the mesh size.

\subsection{Plastic characteristic length calibration}

The calibration of the plastic characteristic length has been done under the hypothesis that $a_{1}=a_{2}$ and $b_{1}=b_{2}$ in Eq. (46). The characteristic plastic length was chosen in the range between $10^{0} \mathrm{~mm}$ and $10^{-4} \mathrm{~mm}$. From the previous calibration procedure it was evident that the elastic characteristic length for this problem had to be smaller or equal than $10^{-2} \mathrm{~mm}$ for the deformation to localize. However, excessively low values of $\beta$ induce the development of a relatively low values of couple stress if compared to the stress, therefore obliging the adoption of high values of the coefficient b in Eq. (46) if we wanted the couple stresses to have an effect on $\sigma_{\text {eq }}$. Thus for the plastic characteristic length calibration, an elastic characteristic length of $10^{-2} \mathrm{~mm}$ has been chosen. Once again, we look at the effects on the global load-displacement graph and to the temperature distribution inside the ASB, which are reported in Figures $6 \mathrm{a}$ and $6 \mathrm{~b}$ respectively.

In Figure 6a it can be observed that the load-displacement curves are bounded between the curves obtained for $l_{p l}=10^{0} \mathrm{~mm}$ and $l_{p l}=10^{-4} \mathrm{~mm}$, 


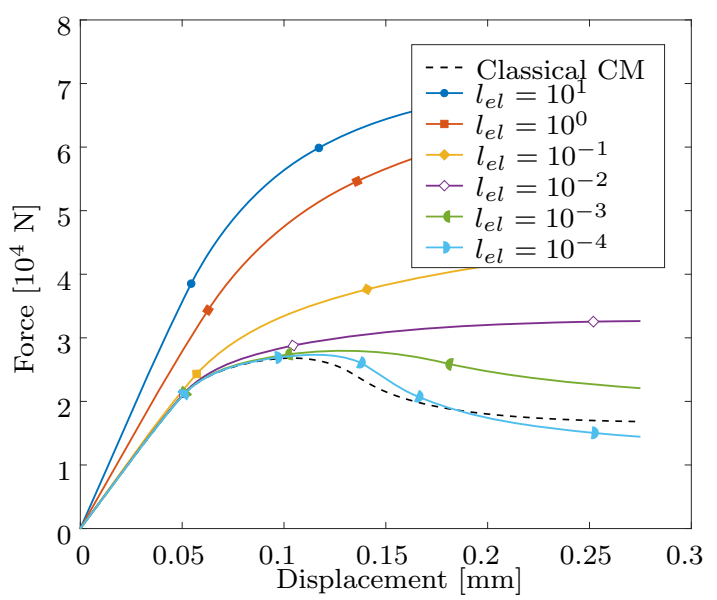

(a)

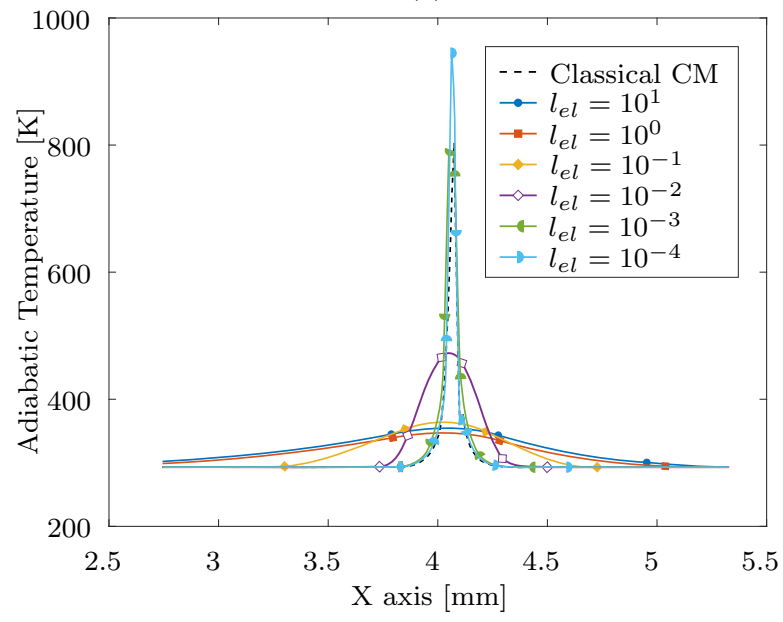

(b)

Fig. 4: Effects of the characteristic elastic length on the load-displ graph (a) and on the temperature distribution (b). Values of the characteristic lengths are given in millimeters.

which represent the asymptotic results for larger or smaller characteristic plastic lengths respectively. This behavior suggests that the plastic development is simultaneously driven by both strain and wryness in case the characteristic plastic length belongs to the range $\approx\left[10^{-1} \mathrm{~mm} \div 10^{-3} \mathrm{~mm}\right]$, and that it is purely dominated by strain or wryness only in case the characteristic plastic length falls respectively before or after this domain.

If we look at the temperature distribution across the ASB in Figure 6b, it shows an increasing peak of predicted temperature at the center of the shear band, and a narrower localization of the thermal field with a decreasing plas- 


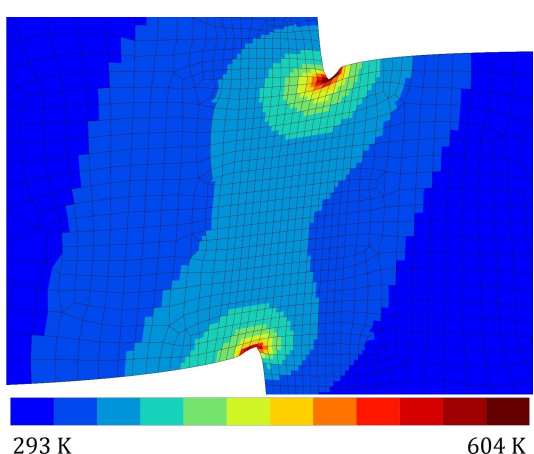

(a) $l_{e l}=10^{0} \mathrm{~mm}$

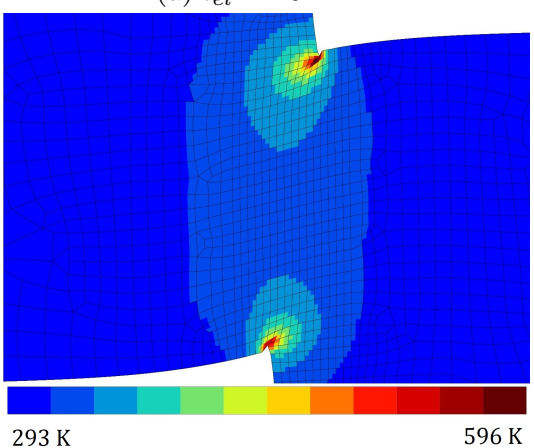

(c) $l_{e l}=10^{-2} \mathrm{~mm}$

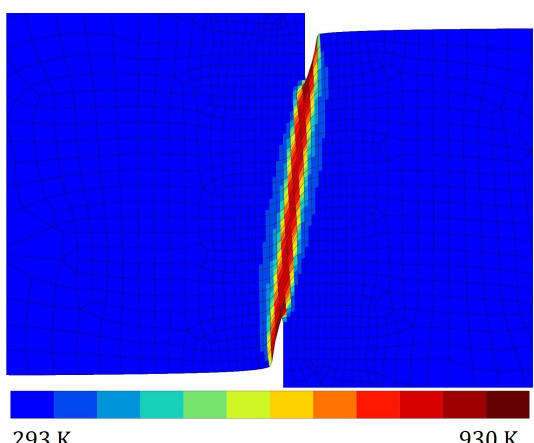

(e) $l_{e l}=10^{-4} \mathrm{~mm}$

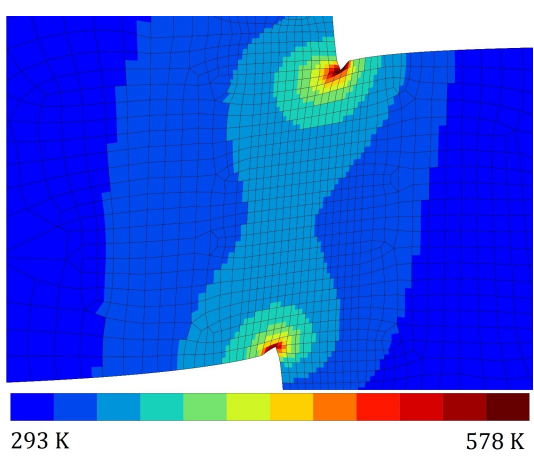

(b) $l_{e l}=10^{-1} \mathrm{~mm}$

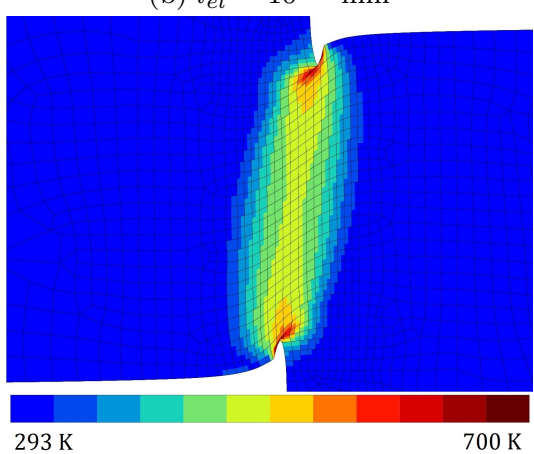

(d) $l_{e l}=10^{-3} \mathrm{~mm}$

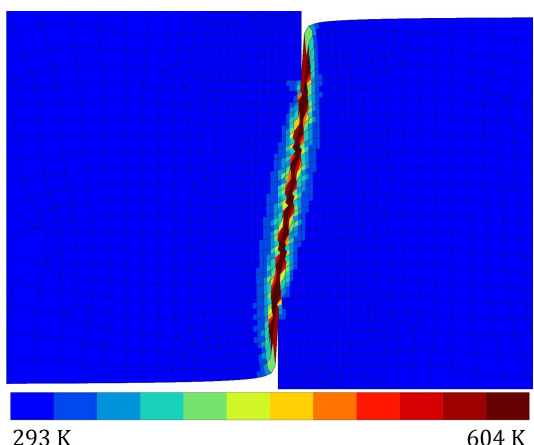

(f) Classical CM

Fig. 5: Temperature distribution in a Hat-Shaped specimen using the Cosserat framework with different values of $\beta$ compared to the Classical CM result. 


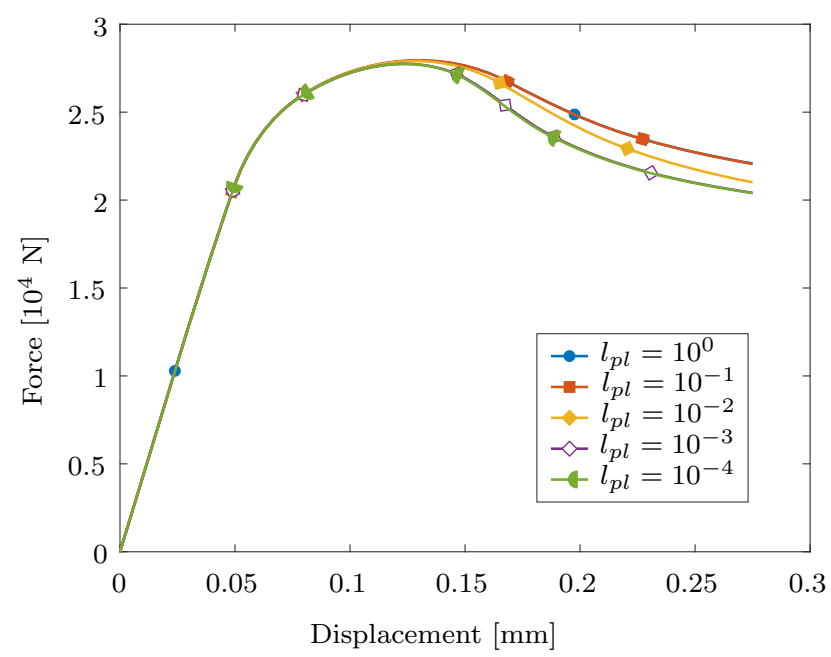

(a)

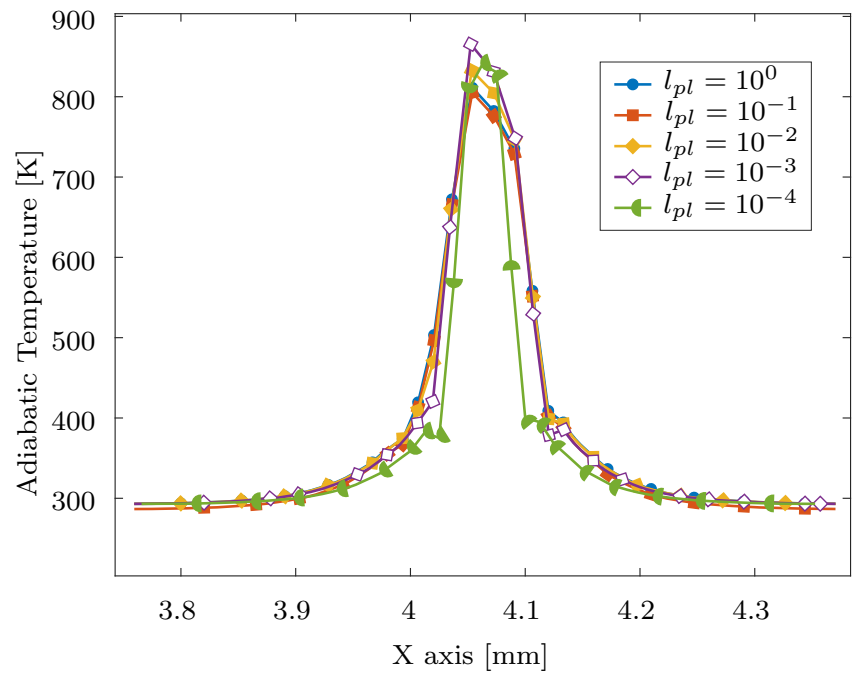

(b)

Fig. 6: Effects of the characteristic plastic length on the load-displ graph (a) and on the temperature distribution (b). Values of the characteristic lengths are given in millimeters.

tic characteristic length. The maximum difference between the peaks of the temperatures belonging to the different plastic characteristic lengths measures $65 \mathrm{~K}$. This difference lies well beyond the resolution of instruments, like thermocouples or thermal camera, thus the effect of different plastic characteristic lengths could be probed. Nonetheless, $65 \mathrm{~K}$ of difference is still a relative low value if compared with the total produced temperature, that is around $500 \mathrm{~K}$. 
This indicates a relative weaker influence of the characteristic plastic length on the developed thermal field if compared with the effects produced by different values of elastic characteristic lengths on the thermal fields.

\section{Conclusion}

Simulations of manufacturing processes can provide valuable information that help optimizing machined materials and machining benches. Furthermore, the knowledge related to the deformation history of the material is a key factor in addressing the post-processed status of the medium, for example in terms of residual stresses. In order to achieve more reliable simulations of these processes, we proposed the adoption of the Cosserat model as description of the medium behavior. A thermodynamically-consistent geometrically linearized formulation of the Cosserat theory was proposed in Section 2, where a thermoelasto-visco-plastic framework was provided for the material characterization through the definition of visco-plastic potential, Helmholtz free energy and dissipation potential.

Using a purely-softening plastic behavior, the Cosserat medium was first proven to provide mesh-independent results, then the TANH flow-stress model was implemented, and it was used to describe an example of the calibration procedure of the elastic and plastic characteristic lengths using a hat-shaped specimen. The calibration of the elastic characteristic length was done by comparing the global load-displacement graph, and, for the specific plastic model used here, its values was found to be in the range of microns, that is comparable to grain size of the Titanium alloy used for the simulation [16]. By fixing the value of the elastic characteristic length to be equal to $10^{-2} \mathrm{~mm}$, the calibration of the plastic characteristic length was performed once again by comparing the load-displacement graphs. The analysis suggested the existence of a range for the plastic characteristic length in which the contribution of plastic strain and plastic wryness were playing a comparable role. Outside these limits the plastic developments were fully dominated by the plastic components of one of the two deformation measures. However, finally the elastic characteristic length was found to have greater effects than the plastic ones.

Nonetheless, since the effects of the plastic characteristic length are highlighted at large plastic deformation, it is advisable to use a large deformation theory for the Cosserat medium. This remains the topic for the future research. For future research it is also foreseen the adoption of a physically-based material plastic law, ideally dislocation-based, and the validation of the Cosserat model for more complex manufacturing operations, such as machining or orthogonal cutting.

Acknowledgements This project has received funding from the European Union's Marie Skłodowska-Curie Action (MSCA) Innovative Training Network (ITN) H2020-MSCA-ITN2017 under the grant agreement $\mathrm{N}^{\mathrm{Q}} 764979$. 


\section{A Derivation of $\dot{T}$}

The derivation of the temperature rate can be derived starting from the definition of the entropy from Eq. (27) and assuming the Helmholtz free energy function as in Eq. (21):

$$
\begin{aligned}
& \eta=-\frac{1}{2} \mathbf{e}^{e}: \frac{\partial \boldsymbol{\Lambda}}{\partial T}: \stackrel{\mathbf{e}}{\sim}^{e}-\frac{1}{2} \mathbf{k}^{e}: \frac{\partial \mathbf{C}}{\partial T}:{\underset{\mathbf{k}}{e}}^{e}+\left(T-T_{0}\right) \frac{\partial \mathbf{P}}{\partial T}: \stackrel{\mathbf{e}}{\sim}^{e} \\
& +\underset{\sim}{\mathbf{P}}: \stackrel{\mathbf{e}}{e}^{e}+\left(T-T_{0}\right) \frac{\partial \mathbf{P}_{k}}{\partial T}:{\underset{\sim}{\mathbf{k}}}^{e}+{\underset{\sim}{\mathbf{P}_{k}}: \mathbf{k}^{e}}^{\mathbf{k}} \\
& +\frac{C_{\varepsilon}}{T_{0}}\left(T-T_{0}\right)-\frac{\partial \psi_{\alpha}}{\partial T}+\underset{\sim}{\boldsymbol{\sigma}}: \frac{\partial \mathbf{e}^{t h}}{\partial T}+\underset{\sim}{\boldsymbol{\mu}}: \frac{\partial \mathbf{k}^{t h}}{\partial T} ;
\end{aligned}
$$

then, by then taking the time variation of the entropy:

$$
\dot{\eta}=\frac{\partial \eta}{\partial \mathbf{\sim}^{e}}:{\underset{\sim}{\dot{e}^{e}}}^{e} \frac{\partial \eta}{\partial \mathbf{k}^{e}}: \underset{\sim}{\dot{\mathbf{k}}}{ }^{e}+\frac{\partial \eta}{\partial T} \dot{T}+\frac{\partial \eta}{\partial \alpha} \dot{\alpha}
$$

where:

$$
\begin{aligned}
& \frac{\partial \eta}{\partial \stackrel{\sim}{e}^{e}}=-{\underset{\sim}{e}}^{e}: \frac{\partial \underset{\boldsymbol{\Lambda}}{\approx}}{\partial T}+\underset{\sim}{\mathbf{P}}+\left(T-T_{0}\right) \frac{\partial \mathbf{P}}{\partial T}+\frac{\partial \mathbf{e}^{t h}}{\partial T}: \underset{\boldsymbol{\Lambda}}{\approx} \\
& \frac{\partial \eta}{\partial \mathbf{k}^{e}}=-{\underset{\sim}{\mathbf{k}^{e}}}^{e}: \frac{\partial \mathbf{C}}{\partial T}+\underset{\sim}{\mathbf{P}_{k}}+\left(T-T_{0}\right) \frac{\partial \mathbf{P}_{k}}{\partial T}+\frac{\partial \mathbf{k}^{t h}}{\partial T}: \underset{\mathbf{C}}{\approx} ; \\
& \frac{\partial \eta}{\partial \alpha}=-\frac{\partial^{2} \psi_{\alpha}}{\partial T \partial \alpha} ;
\end{aligned}
$$

and:

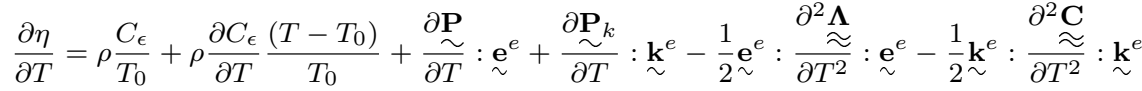

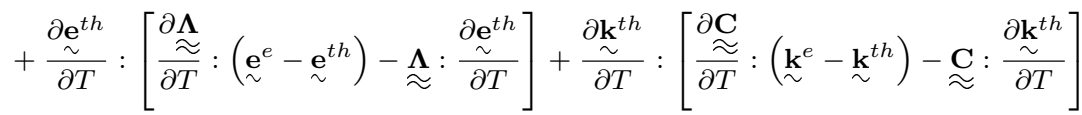

$$
\begin{aligned}
& +\frac{\underset{\mathbf{P}}{\partial T}}{\partial T}:{\underset{\sim}{e}}^{e}+\frac{\mathbf{P}_{k}}{\partial T}:{\underset{\sim}{\mathbf{k}}}^{e}+\left(T-T_{0}\right)\left(\frac{\partial^{2} \underset{\mathbf{P}}{\sim}}{\partial T^{2}}:{\underset{\sim}{e}}^{e}+\frac{\partial^{2} \mathbf{P}_{k}}{\partial T^{2}}:{\underset{\mathbf{k}}{ }}^{e}\right)-\frac{\partial^{2} \psi_{\alpha}}{\partial T^{2}} ;
\end{aligned}
$$

and by plugging them it into the heat Equation Eq. (33), we obtain:

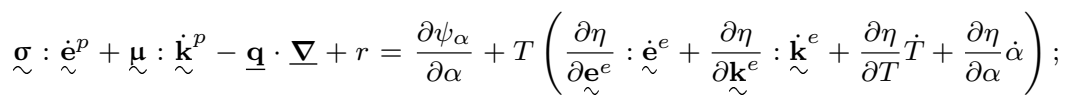

from which the temperature rate can be evaluated as:

$$
\dot{T}=\frac{\underset{\sim}{\boldsymbol{\sigma}}: \dot{\mathbf{e}}^{p}+\underset{\sim}{\boldsymbol{\mu}}: \dot{\mathbf{k}}^{p}-\underline{\mathbf{q}} \cdot \underset{\sim}{\boldsymbol{\nabla}}+r-\frac{\partial \psi_{\alpha}}{\partial \alpha}-T\left(\underset{\sim}{\left.\frac{\partial \eta}{\partial \mathbf{e}^{e}}: \dot{\mathbf{e}}^{e}+\frac{\partial \eta}{\partial \mathbf{w}^{e}}: \dot{\sim}^{e}+\frac{\partial \eta}{\partial \alpha} \dot{\alpha}\right)}\right.}{T \frac{\partial \eta}{\partial T}}
$$

The one reported in Eq. (67) is an expression of the temperature rate which contains many terms whose magnitude is well below the magnitude of the larger terms as $\underset{\sim}{\sigma}: \mathbf{e}^{p}$ for example, therefore assumptions were made on some of the quantities populating Eq. (67) in order to make it usable, and here follows the list of hypotheses: 
Parameter Order of Magnitude

\begin{tabular}{|c|c|}
\hline$\underset{\sim}{\sigma}: \dot{\boldsymbol{e}}^{p}$ & $10^{11}\left[\mathrm{~J} / \mathrm{m}^{3} \cdot \mathrm{s}\right]$ \\
\hline$\underset{\sim}{\mu}: \dot{\mathbf{k}}^{p}$ & $10^{10}\left[\mathrm{~J} / \mathrm{m}^{3} \cdot \mathrm{s}\right]$ \\
\hline$\rho C_{\varepsilon}$ & $10^{10}\left[\mathrm{~J} / \mathrm{m}^{3} \cdot \mathrm{K}\right]$ \\
\hline$\frac{\partial \psi_{\alpha}}{\partial \alpha}$ & $10^{10}\left[\mathrm{~J} / \mathrm{m}^{3}\right]$ \\
\hline$\underset{\sim}{\mathbf{P}}:{\underset{\sim}{\mathbf{e}}}^{e}$ & $10^{6}\left[\mathrm{~J} / \mathrm{m}^{3} \cdot \mathrm{s} \cdot \mathrm{K}\right]$ \\
\hline$\underset{\sim}{\sigma}: \underset{\sim}{\Gamma}$ & $10^{6}\left[\mathrm{~J} / \mathrm{m}^{3} \cdot \mathrm{K}\right]$ \\
\hline$\frac{\partial^{2} \psi_{\alpha}}{\partial T^{2}}$ & $10^{-1}\left[\mathrm{~J} / \mathrm{m}^{3} \cdot \mathrm{K}^{2}\right]$ \\
\hline$\frac{\partial^{2} \psi_{\alpha}}{\partial T \partial p}$ & $10^{-1}\left[\mathrm{~J} / \mathrm{m}^{3} \cdot \mathrm{K}\right]$ \\
\hline
\end{tabular}

Table 3: Order of magnitudes of the terms in Eq. (67).

$-\underline{\mathbf{q}}=\mathrm{r}=0$

$-\frac{\partial \boldsymbol{\Lambda}}{\partial T}=\frac{\partial \mathbf{C}}{\partial T}=0$

$-\frac{\partial C_{\varepsilon}}{\partial T}=0$

$-\psi_{\alpha}=\psi_{\alpha}(\alpha, T) \Longrightarrow \frac{\psi_{\alpha}}{{\underset{\sim}{\mathbf{e}^{e}}}^{e}}=\frac{\psi_{\alpha}}{\mathbf{k}^{e}}=0 ;$

$-\mathrm{T}=500 \mathrm{~K}$

$-\underset{\sim}{\boldsymbol{\Gamma}}=\Gamma \underset{\sim}{\mathbf{I}}=10^{-5} \mathrm{~K}^{-1} ; \quad \underset{\sim}{\boldsymbol{\Gamma}} k=\underset{\sim}{0} \mathrm{~K}^{-1} \mathrm{~m}^{-1} ;$

$-\frac{\partial \boldsymbol{\Gamma}}{\partial T}=\frac{\partial \boldsymbol{\Gamma}_{k}}{\partial T}=0$

$-\left|\mathbf{e}^{e}\right|=10^{-3}$

$-\left|{\underset{\sim}{\dot{e}}}^{e}\right|=10^{-1} \mathrm{~s}^{-1} ;$

$-\left|\dot{\mathbf{e}}^{p}\right|=10^{3} \mathrm{~s}^{-1} ;$

$-\left|\mathbf{k}^{e}\right|=10^{-2} \mathrm{~m}^{-1}$

$-|\underset{\sim}{\mathbf{k}}|=10^{-4} \mathrm{~m}^{-1} \mathrm{~s}^{-1}$

$-\left|\dot{\sim}^{p}\right|=10^{2} \mathrm{~m}^{-1} \mathrm{~s}^{-1} ;$

$-|\underset{\sim}{\sigma}|=10^{8} \mathrm{~Pa} ;$ 
$-\underset{\sim}{\boldsymbol{\mu}} \mid=10^{8} \mathrm{~Pa} \mathrm{~m}$

Furthermore, by assuming the material elastic and plastic models to be the ones described in Tables 1 and 2, the terms in Eq. (67) have the orders of magnitudes listed in Table 3.

From the comparison of the order of magnitudes of the terms in Eq. (67) that are presented in Table 3, the temperature flow rule can assume the following form:

$$
\dot{T}=\frac{\underset{\sim}{\boldsymbol{\sigma}}: \dot{\mathbf{e}}^{p}+\underset{\sim}{\boldsymbol{\mu}}: \dot{\mathbf{k}}^{p}-\frac{\partial \psi_{\alpha}}{\partial \alpha} \dot{\alpha}+T \frac{\partial^{2} \psi_{\alpha}}{\partial T \partial \alpha} \dot{\alpha}}{\rho T\left[\frac{C_{\varepsilon}}{T_{0}}+\frac{\partial C_{\varepsilon}}{\partial T} \frac{\left(T-T_{0}\right)}{T_{0}}\right]-\frac{\partial^{2} \psi_{\alpha}}{\partial T^{2}} T} ;
$$

\section{References}

1. Z-set. non-linear material and structure analysis suite. http://zset-software.com. Accessed: 2020-03-02

2. Aldakheel, F., Miehe, C.: Coupled thermomechanical response of gradient plasticity. International Journal of Plasticity 91, 1-24 (2017). DOI 10.1016/j.ijplas.2017.02.007. URL http://dx.doi.org/10.1016/j.ijplas.2017.02.007

3. Ask, A., Forest, S., Appolaire, B., Ammar, K.: A Cosserat-phase-field theory of crystal plasticity and grain boundary migration at finite deformation. Continuum Mechanics and Thermodynamics (2018). DOI 10.1007/s00161-018-0727-6. URL https://doi. org/10.1007/s00161-018-0727-6

4. Bauer, S., Dettmer, W.G., Perić, D., Schäfer, M.: Micropolar hyper-elastoplasticity: constitutive model, consistent linearization, and simulation of $3 \mathrm{D}$ scale effects. International Journal for Numerical Methods in Engineering 91(1), 39-66 (2012). DOI 10.1002/nme.4256. URL http://doi.wiley.com/10.1002/nme.4256

5. de Borst, R.: Simulation of strain localization: a reappraisal of the cosserat continuum. Engineering Computations 8(4), 317-332 (1991). DOI 10.1108/eb023842. URL http: //www. emeraldinsight.com/doi/10.1108/eb023842

6. de Borst, R.: A generalisation of theory for polar continua. Computer Methods in Applied Mechanics and Engineering 103(3), 347-362 (1993). DOI 10.1016/0045-7825(93) 90127-J. URL https://linkinghub.elsevier.com/retrieve/pii/004578259390127J

7. de Borst, R., Sluys, L.J.: Localisation in a Cosserat continuum under static and dynamic loading conditions. Computer Methods in Applied Mechanics and Engineering 90(1-3), 805-827 (1991). DOI 10.1016/0045-7825(91)90185-9

8. Cahuc, O., Darnis, P., Laheurte, R.: Mechanical and Thermal Experiments in Cutting Process for New Behaviour Law. International Journal of Forming Processes 10(2), 235-269 (2007). DOI 10.3166/ijfp.10.235-269. URL http://ijfp.revuesonline.com/ article.jsp?articleId $=10126$

9. Calamaz, M., Coupard, D., Girot, F.: A new material model for $2 \mathrm{D}$ numerical simulation of serrated chip formation when machining titanium alloy Ti-6Al-4V. International Journal of Machine Tools and Manufacture 48(3-4), 275-288 (2008). DOI 10.1016/j. ijmachtools.2007.10.014

10. Chaboche, J.: Constitutive equations for cyclic plasticity and cyclic viscoplasticity. International Journal of Plasticity 5(3), 247-302 (1989). DOI 10.1016/0749-6419(89)90015-6. URL https://linkinghub.elsevier.com/retrieve/pii/0749641989900156

11. Chambon, R., Caillerie, D., El Hassan, N.: One-dimensional localisation studied with a second grade model. European Journal of Mechanics, A/Solids 17(4), 637-656 (1998). DOI 10.1016/S0997-7538(99)80026-6

12. Chen, G., Ren, C., Yang, X., Jin, X., Guo, T.: Finite element simulation of highspeed machining of titanium alloy $(\mathrm{Ti}-6 \mathrm{Al}-4 \mathrm{~V})$ based on ductile failure model. The International Journal of Advanced Manufacturing Technology 56(9-12), 1027-1038 (2011). DOI 10.1007/s00170-011-3233-6. URL http://link.springer.com/10.1007/ s00170-011-3233-6

13. Cheng, C., Mahnken, R.: A multi-mechanism model for cutting simulations based on the concept of generalized stresses. Computational Materials Science 100(PB), 144-158 (2015). DOI 10.1016/j.commatsci.2014.12.028 
14. Cosserat, E., Cosserat, F.: Theorie des corps deformables. A. Hermann et fils (1909)

15. De Borst, R., Mühlhaus, H.B.: Gradient-dependent plasticity: Formulation and algorithmic aspects. International Journal for Numerical Methods in Engineering 35(3), 521-539 (1992). DOI 10.1002/nme.1620350307. URL http://doi.wiley.com/10.1002/ nme. 1620350307

16. Dong, F., Wang, X., Yang, Q., Liu, H., Xu, D., Sun, Y., Zhang, Y., Xue, R., Krishnaswamy, S.: In-situ measurement of Ti-6Al-4V grain size distribution using laserultrasonic technique. Scripta Materialia 154, 40-44 (2018). DOI 10.1016/j.scriptamat. 2018.05.009. URL https://doi.org/10.1016/j.scriptamat.2018.05.009

17. Fleck, N.A., Hutchinson, J.W.: Strain Gradient Plasticity. Advances in Applied Mechanics 33(C), 295-361 (1997). DOI 10.1016/S0065-2156(08)70388-0. URL http: //linkinghub.elsevier.com/retrieve/pii/S0065215608703880

18. Forest, S., Barbe, F., Cailletaud, G.: Cosserat modelling of size effects in the mechanical behaviour of polycrystals and multi-phase materials. International Journal of Solids and Structures 37(46-47), 7105-7126 (2000). DOI 10.1016/S0020-7683(99) 00330-3. URL papers://82d09eba-ea7e-42aa-9e88-31cb27a3a70e/Paper/p383https: //linkinghub.elsevier.com/retrieve/pii/S0020768399003303

19. Forest, S., Sievert, R.: Elastoviscoplastic constitutive frameworks for generalized continua. Acta Mechanica 160(1-2), 71-111 (2003). DOI 10.1007/s00707-002-0975-0

20. Forest, S., Sievert, R.: Nonlinear microstrain theories. International Journal of Solids and Structures 43(24), 7224-7245 (2006). DOI 10.1016/j.ijsolstr.2006.05.012

21. González, D., Alkorta, J., Martínez-Esnaola, J.M., Gil Sevillano, J.: Numerical analysis of the indentation size effect using a strain gradient crystal plasticity model. Computational Materials Science 82, 314-319 (2014). DOI 10.1016/j.commatsci.2013.10.004. URL http://dx.doi.org/10.1016/j.commatsci.2013.10.004

22. Grammenoudis, P., Tsakmakis, C.: Hardening rules for finite deformation micropolar plasticity: Restrictions imposed by the second law of thermodynamics and the postulate of Il'iushin. Continuum Mechanics and Thermodynamics 13(5), 325-363 (2001). DOI $10.1007 / \mathrm{s} 001610100055$

23. Guo, Y.B., Wen, Q., Woodbury, K.A.: Dynamic material behavior modeling using internal state variable plasticity and its application in hard machining simulations. Journal of Manufacturing Science and Engineering, Transactions of the ASME 128(3), 749-759 (2006). DOI 10.1115/1.2193549

24. He, L., Su, H., Xu, J., Zhang, L.: Simulation analysis of the influence of dynamic flow stress behavior on chip formation. International Journal of Advanced Manufacturing Technology 95(5-8), 2301-2313 (2018). DOI 10.1007/s00170-017-1275-0

25. Hor, A., Morel, F., Lebrun, J.L., Germain, G.: Modelling, identification and application of phenomenological constitutive laws over a large strain rate and temperature range. Mechanics of Materials 64, 91-110 (2013). DOI 10.1016/j.mechmat.2013.05.002. URL http://dx.doi.org/10.1016/j.mechmat.2013.05.002

26. Huang, J., Kalaitzidou, K., Sutherland, J.W., Milligan, W.W., Aifantis, E.C., Sievert, R., Forest, S.: Gradient Plasticity : Implications to Chip Formation in Machining (2001)

27. Jebahi, M., Cai, L., Abed-Meraim, F.: Strain gradient crystal plasticity model based on generalized non-quadratic defect energy and uncoupled dissipation. International Journal of Plasticity 126(April 2019), 102617 (2019). DOI 10.1016/j.ijplas.2019.10.005. URL https://doi.org/10.1016/j.ijplas.2019.10.005

28. Joshi, S.S., Melkote, S.N.: An Explanation for the Size-Effect in Machining Using Strain Gradient Plasticity. Journal of Manufacturing Science and Engineering 126(4), 679 (2004). DOI 10.1115/1.1688375. URL http://manufacturingscience. asmedigitalcollection . asme .org/article. aspx?articleid=1449147

29. Khoei, A.R., Yadegari, S., Biabanaki, S.O.: 3D finite element modeling of shear band localization via the micro-polar Cosserat continuum theory. Computational Materials Science 49(4), 720-733 (2010). DOI 10.1016/j.commatsci.2010.06.015. URL http: //dx.doi.org/10.1016/j.commatsci.2010.06.015

30. Koiter, W.T.: Couple Stresses in the Theory of Elasticity, I \& II. Philosophical Transactions of the Royal Society of London B 67, 17-44 (1964)

31. Kratochvíl, J., Labbé, E., Rey, C., Yang, S.: On physically motivated mesoscale Cosserat model of shear band formation. Scripta Materialia 41(7), 761-766 (1999). DOI 10.1016/ S1359-6462(99)00214-6 
32. Lele, S.P., Anand, L.: A large-deformation strain-gradient theory for isotropic viscoplastic materials. International Journal of Plasticity 25(3), 420-453 (2009). DOI 10.1016/j.ijplas.2008.04.003. URL http://dx.doi.org/10.1016/j.ijplas.2008.04.003

33. Li, J.C., Chen, X.W., Chen, G.: Numerical simulations on adiabatic shear behaviour of 921A steel pure shear hat-shaped specimens. WIT Transactions on the Built Environment 113, 323-334 (2010). DOI 10.2495/SU100281

34. List, G., Sutter, G., Bi, X.F., Molinari, A., Bouthiche, A.: Strain, strain rate and velocity fields determination at very high cutting speed. Journal of Materials Processing Technology 213(5), 693-699 (2013). DOI 10.1016/j.jmatprotec.2012.11.021

35. Marchand, A., Duffy, J.: An experimental study of the formation process of adiabatic shear bands in a structural steel. Journal of the Mechanics and Physics of Solids 36(3), 251-283 (1988). DOI 10.1016/0022-5096(88)90012-9. URL http: //linkinghub.elsevier.com/retrieve/pii/0022509688900129

36. Molinari, A., Soldani, X., Miguélez, M.H.: Adiabatic shear banding and scaling laws in chip formation with application to cutting of Ti-6Al-4V. Journal of the Mechanics and Physics of Solids 61(11), 2331-2359 (2013). DOI 10.1016/j.jmps.2013.05.006. URL http://dx.doi.org/10.1016/j.jmps.2013.05.006

37. Mühlhaus, H.B., Vardoulakis, I.: The thickness of shear bands in granular. Géotechnique 37(3), 271-283 (1987). DOI 10.1680/geot.1987.37.3.271

38. Neff, P.: A finite-strain elastic-plastic Cosserat theory for polycrystals with grain rotations. International Journal of Engineering Science 44(8-9), 574-594 (2006). DOI 10.1016/j.ijengsci.2006.04.002

39. Nguyen, G.D., Korsunsky, A.M., Belnoue, J.P.: A nonlocal coupled damage-plasticity model for the analysis of ductile failure. International Journal of Plasticity 64, 56-75 (2015). DOI 10.1016/j.ijplas.2014.08.001. URL http://dx.doi.org/10.1016/j.ijplas . 2014.08 .001

40. Pamin, J., Wcisło, B., Kowalczyk-Gajewska, K.: Gradient-enhanced large strain thermoplasticity with automatic linearization and localization simulations. Journal of Mechanics of Materials and Structures 12(1), 123-146 (2017). DOI 10.2140/jomms.2017.12.123. URL http://msp.org/jomms/2017/12-1/p08.xhtml

41. Peirs, J., Verleysen, P., Degrieck, J., Coghe, F.: The use of hat-shaped specimens to study the high strain rate shear behaviour of Ti-6Al-4V. International Journal of Impact Engineering 37(6), 703-714 (2010). DOI 10.1016/j.ijimpeng.2009.08.002. URL http: //dx.doi.org/10.1016/j.ijimpeng.2009.08.002

42. Poole, W., Ashby, M., Fleck, N.: Micro-hardness of annealed and work-hardened copper polycrystals. Scripta Materialia 34(4), 559-564 (1996). DOI 10.1016/1359-6462(95) 00524-2. URL http://linkinghub.elsevier.com/retrieve/pii/1359646295005242

43. Rattez, H., Stefanou, I., Sulem, J., Veveakis, M., Poulet, T.: Numerical Analysis of Strain Localization in Rocks with Thermo-hydro-mechanical Couplings Using Cosserat Continuum. Rock Mechanics and Rock Engineering 51(10), 3295-3311 (2018). DOI 10.1007/s00603-018-1529-7

44. Royer, R., Darnis, P., Laheurte, R., Gérard, A., Cahuc, O.: Finite strain gradient plasticity theory for high speed machining. Procedia Engineering 10, 2312-2317 (2011). DOI 10.1016/j.proeng.2011.04.381

45. Sabet, S.A., de Borst, R.: Structural softening, mesh dependence, and regularisation in non-associated plastic flow. International Journal for Numerical and Analytical Methods in Geomechanics 43(13), 2170-2183 (2019). DOI 10.1002/nag.2973

46. Śloderbach, Z., Pająk, J.: Thermodynamic potential of free energy for thermo-elasticplastic body. Continuum Mechanics and Thermodynamics 30(1), 221-232 (2018). DOI 10.1007/s00161-017-0597-3

47. Steinmann, P., William, K.: Localization within the Framework of Micropolar ElastoPlasticity. Advances in Continuum Mechanics pp. 296-313 (1991). DOI 10.1007/ 978-3-642-48890-0_24

48. Stölken, J., Evans, A.: A microbend test method for measuring the plasticity length scale. Acta Materialia 46(14), 5109-5115 (1998). DOI 10.1016/S1359-6454(98)00153-0. URL http://www.edpsciences.org/10.1051/jp4: 2000932http://linkinghub.elsevier.com/retrieve/pii/S1359645498001530 
49. T, B.G.I., Taylor, G.I., Quinney, H.: The latent energy remaining in a metal after cold working. Proceedings of the Royal Society of London. Series A, Containing Papers of a Mathematical and Physical Character 143(849), 307-326 (1934). DOI 10.1098/rspa. 1934.0004

50. Tordesillas, A., Peters, J.F., Gardiner, B.S.: Shear band evolution and accumulated microstructural development in Cosserat media. International Journal for Numerical and Analytical Methods in Geomechanics 28(10), 981-1010 (2004). DOI 10.1002/nag. 343

51. Wang, W.M., Sluys, L.J., De Borst, R.: Viscoplasticity for instabilities due to strain softening and strain-rate softening. International Journal for Numerical Methods in Engineering 40(20), 3839-3864 (1997). DOI 10.1002/(SICI)1097-0207(19971030)40: 20<3839::AID-NME245〉3.0.CO;2-6

52. WANG, X.b.: Adiabatic Shear Localization for Steels Based on Johnson-Cook Model and Second- and Fourth-Order Gradient Plasticity Models. Journal of Iron and Steel Research International 14(5), 56-61 (2007). DOI 10.1016/S1006-706X(07)60075-2

53. Wcisło, B., Pamin, J.: Local and non-local thermomechanical modeling of elastic-plastic materials undergoing large strains. International Journal for Numerical Methods in Engineering 109(1), 102-124 (2017). DOI 10.1002/nme.5280

54. Wu, J., Liu, Z.: Modeling of flow stress in orthogonal micro-cutting process based on strain gradient plasticity theory. International Journal of Advanced Manufacturing Technology 46(1-4), 143-149 (2010). DOI 10.1007/s00170-009-2049-0

55. Ye, G.G., Chen, Y., Xue, S.F., Dai, L.H.: Critical cutting speed for onset of serrated chip flow in high speed machining. International Journal of Machine Tools and Manufacture 86, 18-33 (2014). DOI 10.1016/j.ijmachtools.2014.06.006

56. Yuan, H., Chen, J.: Identification of the intrinsic material length in gradient plasticity theory from micro-indentation tests. International Journal of Solids and Structures 38(46-47), 8171-8187 (2001). DOI 10.1016/S0020-7683(01)00121-4

57. Zheng, Q.S.: Theory of Representations for Tensor Functions-A Unified Invariant Approach to Constitutive Equations. Applied Mechanics Reviews 47(11), 545 (1994). DOI 10.1115/1.3111066. URL http://appliedmechanicsreviews.asmedigitalcollection. asme.org/article.aspx?articleid=1395390 